\title{
Exploring the cognitive infrastructure of communication
}

\author{
J.P. de Ruiter ${ }^{*}, 1,5, \mathrm{a}$, Matthijs L. Noordzij ${ }^{2,4, a}$, Sarah Newman- \\ Norlund $^{2}$, Roger Newman-Norlund ${ }^{2,3}$, Peter Hagoort ${ }^{2,3}$, \\ Stephen C. Levinson ${ }^{1} \&$ Ivan Toni ${ }^{2,3}$
}

${ }^{1}$ Max Planck Institute for Psycholinguistics, Nijmegen, The Netherlands/2F.C. Donders Centre for Cognitive Neuroimaging, Radboud University Nijmegen, The Netherlands $/{ }^{3} \mathrm{Nijmegen}$ Institute for Cognition and Information, Radboud University Nijmegen, The Netherlands/4 Department of Cognitive Psychology and Ergonomics, University of Twente, Enschede, The Netherlands $/{ }^{5}$ Department of Linguistics and Literary Studies. University of Bielefeld, Bielefeld, Germany

Human communication is often thought about in terms of transmitted messages in a conventional code like a language. But communication requires a specialized interactive intelligence. Senders have to be able to perform recipient design, while receivers need to be able to do intention recognition, knowing that recipient design has taken place. To study this interactive intelligence in the lab, we developed a new task that taps directly into the underlying abilities to communicate in the absence of a conventional code. We show that subjects are remarkably successful communicators under these conditions, especially when senders get feedback from receivers. Signaling is accomplished by the manner in which an instrumental action is performed, such that instrumentally dysfunctional components of an action are used to convey communicative intentions. The findings have important implications for the nature of the human communicative infrastructure, and the task opens up a line of experimentation on human communication.

\section{Introduction}

First principles suggest that there is a hidden infrastructure to the use of language and other human conventional communication systems. This infrastructure can

\footnotetext{
${ }^{\star}$ Corresponding author, can be contacted at jan.deruiter@uni-bielefeld.de, or at P.O. Box 100131, 33501 Bielefeld, Germany.

aThese authors contributed equally to this work.
} 
bootstrap learners of a language into their first meanings, which gives us the means to constantly learn new words, it can help us disambiguate utterances that are nearly always multiply ambiguous, and it can help locate a definite message in utterances that are vague or general. Grice (1957) in his well-known 'theory of meaning' suggested that this infrastructure was the essential ability to recognize a signal as being designed to be interpretable - in fact 'meaning' in a general sense essentially consists of recognizing the signaler's intentions. Levinson (1995) suggested that the ability to engage in this reflexive reasoning about each other's intentions constitutes a very special capacity, an interactional intelligence, which is one of the hallmarks of human cognition. This paper is an attempt to explore this underlying capacity by isolating it from the superstructure of conventional linguistic signs that normally accompanies it (see also Galantucci, 2005; Garrod, Fay, Lee, Oberlander, \& Macleod, 2007; Healey, Swoboda, Umata, \& King, 2007).

It is not always immediately obvious why human communication is such a complex enterprise, or why it requires special cognitive abilities. If one were to take Shannon's (1948) mathematical theory of communication as a conceptual starting point, communication appears - on first glance - to be relatively straightforward. In order for a sender to get information across to a receiver, the sender encodes information, transmits it to the other side using a physical medium (e.g., sound waves through the air), the receiver decodes this information, and communication has been achieved. The main challenge to be tackled in the Shannon framework is overcoming the problem of signal degradation. Creating redundancy in the signal makes the communication process less susceptible to the detrimental effects of noise. Shannon's work is relevant for one important aspect of communication, namely data transmission. For instance, it has been shown that speech signals contain redundancy, and this redundancy enables listeners to retrieve phonemes from a speech stream under suboptimal circumstances like noisy environments (Repp \& Liberman, 1987).

However, data transmission is not where the real complexity of human-human interaction resides. What is much less understood is how receivers are able to extract the communicative intention that motivated the sender of a signal to send it, i.e., the meaning of the transferred information. This problem is generally referred to as intention recognition (see e.g., Levelt, 1989, p. 59; Wharton, 2003). Consequently, senders have to produce signals in such a way that the receiver has a reasonable chance to do successful intention recognition. This counterpart of intention recognition has been called recipient design (Sacks \& Schegloff, 1979).

The challenge of mapping communicative intentions on to utterances is at the core of the field of linguistic pragmatics (Levinson, 1983), and relies heavily on the ability to recognize intentions. A compelling example of this is the (often 
successful) use of highly ambiguous definite references such as "the what do you call it" (Enfield, 2003). As Levinson (2006) notes, statistical (e.g., Bayesian) methods are not sufficient to provide a general solution to this problem, because there are many actions whose interpretation are not in line with statistical associations. In ironic utterances, for instance, it is the low probability of the utterance given the communicative intention that makes them interpretable at all. Also, Bayesian methods rely on previous experience and cannot explain the fast and successful interpretation of signal-to-meaning mappings that a receiver has never encountered before.

In order to make it possible for a listener to reconstruct the communicative intention from an utterance, a speaker has to ensure that his utterances are constructed so that it can be expected that the listener can actually retrieve its original communicative intention. This holds not only for linguistic communication, but also for non-linguistic communication of any kind. This ability to communicate without relying on shared conventions is of fundamental importance. It allows human communicators to get across rather elaborate meanings even when the communicative circumstances are sub-optimal (e.g., divers under water, people in noisy factories) or even when they do not share a common language (the classic "tourist asking for directions" scenario). More importantly, this faculty is a crucial enabling factor for the creation and use of linguistic symbols (Tomasello, Carpenter, Call, Behne, \& Moll, 2005). In fact, it seems to provide the essential means for learning and developing a conventional communication system - if you can't grasp an intended meaning without knowing the conventional meaning of a signal, there would be no way to learn it. Consider the case of 'home sign' (Goldin-Meadow, Gelman, \& Mylander, 2005), where a deaf child of hearing parents invents a sign language de novo.

Summarizing, we assume that effective communication involves complex computations at both the sender and the receiver's end. The receiver's problem is one of intention recognition; the sender's problem is one of recipient design.

\section{The role of conventions}

In many situations the computational complexity of communication can be reduced by using previously established conventions (see Lewis, 1968). Instead of performing cumbersome computations of the type 'what would he think I would think if he...' (Clark \& Marshall, 1981), human communicators might simply have learned over time that 'can you tell me the time?' is a not an inquiry into the recipient's abilities, but rather a request to tell the current time, and that it is polite to formulate it that way. While it is plausible that most of the time we can rely 
on communicative conventions, the interactive intelligence needed for recipient design is nevertheless essential for successful communication in general. First, in order to learn new conventions, we need to be able to recognize the communicative intention of a signal the first time we encounter it, i.e., before a convention has been established. Second, we often find ourselves in situations where no communicative conventions exist yet, and still are able to get our intentions recognized. To solve this problem, humans need certain heuristics. Following the lucid overview in Garrod \& Anderson (1987) here, one example of such a heuristic is provided by the coordination game investigated by Schelling (1960). Schelling proposes that people use salience to achieve coordination, on the grounds that what is salient for the communicator is also likely to be salient for their interlocutor. Another heuristic that can be used is precedence (Schiffer, 1972): if a certain form-meaning mapping has been used in the past, it can be used again. A key study of the development of new communicative (and non-linguistic) conventions is Galantucci (2005) (see also Steels, 2006), in which he required participants who were playing a computer game to communicate vital information using a completely novel (and rather noisy) communicative channel. After a number of attempts, subjects were able to develop some kind of conventional signal/meaning mapping and use that convention to their advantage in the game. This study is an intriguing demonstration of how humans can develop new semiotic conventions when they need to. However, we want to stress that interactive intelligence is not only needed in exceptional situations; work on human dialogue (Brennan \& Clark, 1996; Garrod \& Anderson, 1987; Levinson, 1983; Schegloff, 1988) has shown that even ordinary, verbal interaction between people who share the same language requires interactive intelligence to deal with the pervasive ambiguity of communicative signals.

\section{The present study}

As discussed above, there are a priori logical arguments for the view that in order to communicate successfully, people need a special kind of communicative intelligence, and there is experimental evidence for the existence of this capacity in human communicators (Galantucci, 2005). Here, we want to explore these abilities under controlled experimental conditions, using traditional dependent variables such as reaction times. For this purpose, we have developed the Tacit Communication Game (TCG, see De Ruiter, Noordzij, Newman-Norlund, Hagoort, \& Toni, 2007), an experimental paradigm for studying human-human communication, while limiting the possibility of using pre-established communicative conventions. The TCG 
is designed to supplement other well-established linguistic communication tasks such as the Map Task (Anderson, et al., 1991), or the SLOT paradigm (De Ruiter, Rossignol, Vuurpijl, Cunningham, \& Levelt, 2003), and it differs from the task used by Galantucci (2005) in that it is designed to focus on those cognitive processes in individual communicators that are responsible for the development and recognition of newly created conventions. The TCG also enables the study of the nature of those conventions. We use the TCG to address the following questions:

1. How well can senders communicate their intentions to receivers when there are insufficient pre-existing communicative conventions?

2. Do reaction times obtained with this task reflect the processing load related to interactive processes in the sender?

3. What is the relationship between the processing load of senders and receivers?

4. How do senders use the (very limited) expressive means available to them to encode their communicative intentions?

Before reporting on the experiments addressing the above questions, we will first outline the essentials of the Tacit Communication Game.

\section{The Tacit Communication Game}

In this game (or task), there are two subjects, who are seated behind separate computer screens. These two screens are controlled by one central computer. One subject has the role of a sender and the other of a receiver. (For clarity, the male pronoun 'he' will be used for the sender, while the female pronoun 'she' will be used for the receiver.) On the computer screens of both the sender and the receiver, a 3 by 3 grid (the 'playing board') is visible. Below the grid, the geometrical shape that is under the control of the sender is shown, and above the grid there is a geometrical object that is under the control of the receiver. The three possible objects of the sender and receiver are a rectangle, a circle, or a triangle. The participants can move their object from one grid position to another, but only along horizontal and vertical directions. The objects can also be rotated clockwise or counterclockwise, within a given grid position. However, when applied to the circle object, these rotations are not visible.

In a typical communicative trial (other experimental conditions are discussed below), the sender and only the sender will see a goal configuration, which consist of the sender and receiver objects both placed at specific locations in the grid in a specific orientation. An example goal configuration can be seen in Fig. 1. 


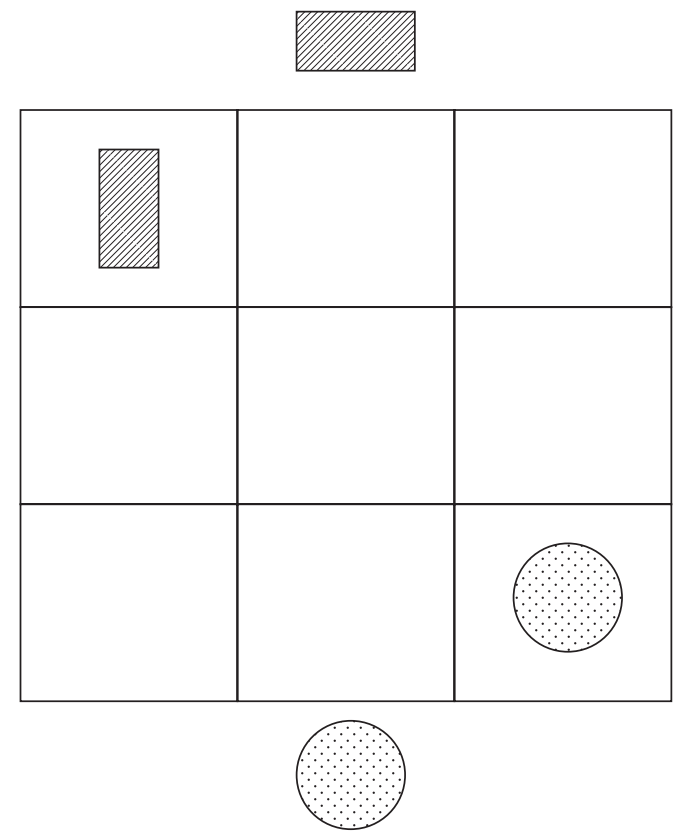

Figure 1. Example goal configuration for TCG

The sender now has to consider and perform two tasks. The first is how to move his own object to the location displayed in the goal configuration (i.e., lower right corner, see Fig. 1). But at the same time, the sender has to also communicate to the receiver where she should place her object (the rectangle), and in which orientation. The only means the sender has available to transmit this information to the receiver is moving around and rotating his own object using a game controller.

The sender is thus required to think of a strategy how to do this. He has unlimited time for doing this, and during this planning time the goal configuration is visible to him. When the sender has finished planning and presses the start button, the goal configuration disappears from the screen, the sender's object (in this case a circle) appears in the center of the grid, and then the sender has five seconds to move around and/or rotate his object and achieve his two goals: communicate to the receiver where and in which orientation she should put her object, and get his own object at the correct location and orientation. After the 5 seconds are over and the sender has finished moving his object around (and the moves have been observed by the receiver) the receiver's object is placed in the center of the grid, and she can now move her object to the location and orientation that she believes is the correct one. A trial is considered to be successful when both sender and receiver have put their objects in the location and orientation as specified in the goal configuration within the allowed time. 
An important aspect of the TCG is that, as in the experiment by Galantucci (2005), it can be assumed that neither the sender nor the receiver have ever been in such a communicative situation before, so there are no pre-established communicative conventions that the subjects can rely on.

\section{Experiment 1}

\subsection{Method}

\subsubsection{Participants}

15 pairs of participants (aged 18-26 years; 20 females, 10 males) were recruited to participate. They were either offered a standard financial payment (€6 per hour) or given credits towards completing a course requirement. Three pairs were excluded from analysis based on a misunderstanding of the instruction or the use auditory communication, which was not allowed during the game, leaving 12 pairs of subjects. These 12 pairs were randomly assigned to one of two trial orders. Trial order was varied for the purpose of counter-balancing for possible order effects.

\subsubsection{Equipment}

The game was played on two 19-inch black computer monitors with Logitech hand-held controllers (see Fig. 2). The buttons used on the hand-held controller were spatially laid out to provide quick and easy learning of spatial movements. Players sat at a long table on opposing sides with the computer in between them each facing their own computer monitor. Due to the length of the table and the position and size of the monitors, the players could not see each other. The game was programmed using Presentation version 9.2 and was run on a Windows XP personal computer.

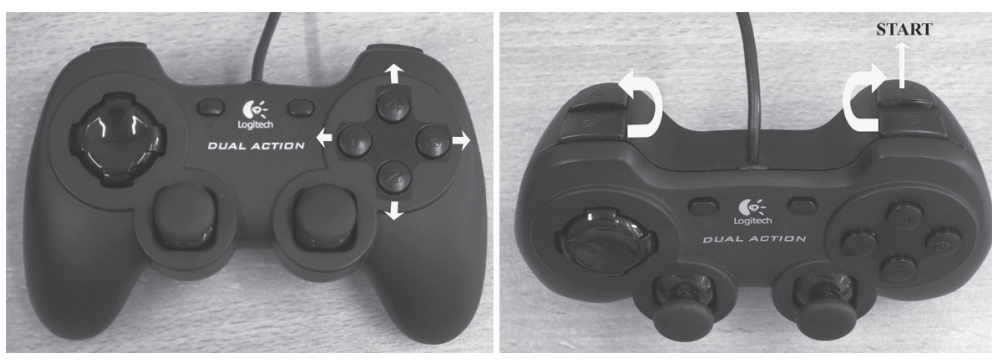

Figure 2. The Game Controller with four spatial buttons to move around the board (indicated in the front view) and with rotation buttons located on top of the controller (left/right, indicated in the top view). There was one start button on top of the controller below the right rotation button 


\subsubsection{Procedure}

The game board consists of a $3 \times 3$ grid of grey interlocking tiles presented on a black background (see Fig. 3). Game pieces were on the same grey tiles and additionally had one of three shapes centered on the tile: circle, rectangle, or triangle. Shapes were blue, red, or yellow, and had an original starting orientation (horizontal rectangle, and point of triangle heading up). Before each trial began and while the goal configuration was on the screen, the sender's shape was located under the game board, and the receiver's shape was located above the game board. When the sender pressed his start button, the goal disappeared from the screen leaving the grey game board and the sender's shape in the center box in its "original" orientation. In all trials, each player had 5 seconds to move. When the sender's time was over, the receiver's shape appeared in the center of the game board. The receiver then had additional time to interpret what she just saw and plan her movements, and subsequently pushed the start button to begin her movement, beginning the 5 second time limit. When the receiver's time expired both players received feedback regarding their accuracy as a team by a red or green box; incorrect or correct.

For the remainder of this paper, the planning time of the sender is defined as the time between the beginning of a trial and the sender pressing the "start" button, and the planning time of the receiver is defined as the time between the end of the last move of the sender and the pressing of the "start button" by the sender.

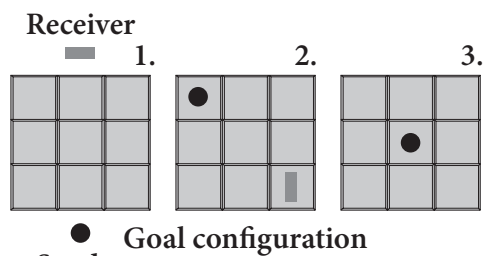

3.

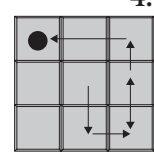

4.

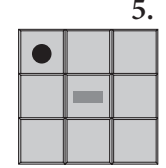

5.

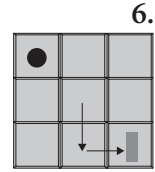

7.

Sender

Figure 3. Sequence of events in a TCG trial

1. Players view their shapes (1500 ms)

2. Player(s) see the goal configuration

3. The sender starts his movement sequence by pressing the start button

4. The sender has $5000 \mathrm{~ms}$ to move the shape around on the game board

5. The receiver begins movements from center tile by pressing the start button (the sender's shape remains on screen in position they left it)

6. The receiver has $5000 \mathrm{~ms}$ to move her shape around in the game board

7. Players receive feedback indicating whether they were correct (green box) or incorrect (red box) in matching the goal configuration (both the sender and the receiver received this feedback, indicating whether they were successful as a team in reproducing the goal configuration)

After being instructed regarding the experimental tasks, participants provided their informed consent. Training was conducted to prepare them to play the game 
together. There were two training sessions before the game began to familiarize the participants with the hand-held controller, the order of events in the game, and the characteristics of the stimuli. After training, written instructions were then provided regarding the game. In all tasks, participants were instructed to perform as accurately as possible.

Individual Training. Each participant first trained individually for 40 trials on how to use the joystick device to move and rotate their shape on the screen. On the game board $(3 \times 3)$, they practiced matching goal configurations using a triangle, to maximize the practice of rotating their object (the triangle has the highest number of possible orientations). Each trial continued until they matched the goal configuration with their shape. When they correctly matched the goal, they received a green box indicating they were right. A subsequent trial immediately followed. There were no time limits for the initial training.

Joint Training. Once participants felt comfortable with the hand-held controller, they performed a practice session involving 20 trials where both players saw the goal configuration and moved to their own location (or did not move because the goal position was in the center). The joint training goal configurations utilized each of the 9 boxes and each of the 3 shapes (circle, rectangle, or triangle). The goal configurations were intermixed with trials where both players either had the same shape or different shapes.

\subsubsection{Materials}

The game consisted of 120 trials. In the first 20 and thwe last 20 trials of game, all players could see the goal configurations and their task was simply to go to their own location and match their own orientation. In the middle 80 trials, the receiver could not see the goal configuration. Players either had the same shapes or had different shapes. Table 1 shows how the trials were distributed in such a way that the same and different shape trials were counterbalanced over the two groups when the receiver did not see the goal.

Table 1. Counterbalancing of conditions over the subjects

\begin{tabular}{|c|c|c|}
\hline Trial Order & Group A & Group B \\
\hline Trials 1-20 & $\begin{array}{l}\text { Both players see goal } \\
\text { Mixed trials }\end{array}$ & $\begin{array}{l}\text { Both players see goal } \\
\text { Mixed trials }\end{array}$ \\
\hline Trials 21-40 & $\begin{array}{l}\text { The receiver doesn't see goal: } \\
\text { Same shapes }\end{array}$ & $\begin{array}{l}\text { The receiver doesn't see goal: } \\
\text { Different shapes }\end{array}$ \\
\hline Trials 41-80 & $\begin{array}{l}\text { The receiver doesn't see goal: } \\
\text { Different shapes }\end{array}$ & $\begin{array}{l}\text { The receiver doesn't see goal: } \\
\text { Same shapes }\end{array}$ \\
\hline Trials $81-100$ & $\begin{array}{l}\text { The receiver doesn't see goal: } \\
\text { Same shapes }\end{array}$ & $\begin{array}{l}\text { The receiver doesn't see goal: } \\
\text { Different shapes }\end{array}$ \\
\hline Trials 101-120 & $\begin{array}{l}\text { Both players see goal; } \\
\text { Mixed trials }\end{array}$ & $\begin{array}{l}\text { Both players see goal } \\
\text { Mixed trials }\end{array}$ \\
\hline
\end{tabular}




\subsection{Results}

The main purpose of Experiment 1 was to investigate whether subjects could perform the TCG task with reasonable success rates. We therefore analyzed the data from trials 21-100, in which the sender saw the goal, but the receiver did not. We defined 'success' of a trial as the receiver putting her object in the correct location and orientation. Overall, $83 \%$ of all trials were successful. This is far above chance levels: there are nine possible locations and varying numbers of possible orientations: One for the circle, two for the rectangle, and four for the triangle shape. This means that when the receiver shape is a circle, the probability of being correct purely by chance is $1 / 9$ th, for the rectangle $1 / 18$ th, and for the triangle $1 / 36$ th. The overall success rate of $83 \%$ over a total of 960 trials is significantly higher than any of these base probabilities (binomial test, $p<.001$ ). ${ }^{1}$

It should be noted here that in piloting Experiment 2 (see below) we noticed that one dyad used a clever way to communicate using the clicking noises of the game controller for communicative purposes. Although the experimenters did not notice such strategies occurring during any of the trials in Experiment 1, it cannot be completely ruled out that there had been some form of acoustic "leakage" that escaped their attention. Since we took measures against any form of acoustic leakage in Experiments 2 and 3 by introducing noise resistant headphones and ear-plugs, and since the success rates from those experiments were very similar to the results in this experiment, we assume that the conclusions from Experiment 1 are valid, and that the theoretical possibility of undetected acoustic leakage played no, or at most only a negligible, role in the reported communicative success rates.

Both receivers and senders were faster (in terms of planning time) and more successful with the same-shape trials than with the different-shape trials. A paired t-test comparing the averages of the variables planning time of sender, planning time of receiver and success within each dyad shows that for planning time of sender, the average for same-shape trials was $4148 \mathrm{~ms}$, and for different-shape trials $5245 \mathrm{~ms}$ $(t(11)=3.015, p<.05)$. For planning time of receiver the average was $1089 \mathrm{~ms}$ for same-shape trials, and $1443 \mathrm{~ms}$ for different-shape trials $(t(11)=4.077, p<.01)$. For success (as a proportion) the average was .90 in the same-shape condition and .76 in the different-shape condition $(t(1)=5.797, p>.001)$.

The underlying cause for this difference between same-shape and differentshape trials was the difficulty in communicating the orientation of objects, which was never a problem when the objects were identical. Some combinations of objects, however, lead to difficulties. For instance, indicating a vertical orientation using a circle is difficult because circles cannot be oriented vertically. Similarly, although a rectangle can be oriented horizontally and vertically, these two orientations cannot be used to indicate, for example, that a triangle (which has four possible orientations) is pointing to the right and not to the left. In those trials 
where the indication of orientation was problematic because the sender's object had fewer possible orientations than the receiver's object (we will call these trials 'hard' from here on), the success rate was $67 \%$. This is significantly lower than the overall success rate ( $z$-test for testing independent proportions, $p<.001$ ), but it is still far above chance levels (binomial test, $n=240, p<.0001$ ).

The main result from Experiment 1 is that human subjects can successfully perform the TCG. In the next experiment, we investigated whether or not feedback (in the form of the receiver's behavior) that senders had access to in experiment 1 facilitated the communication. We also performed a qualitative analysis to find out how the subjects used the limited means provided by the TCG to communicate location and orientation to their interlocutor.

\section{Experiment 2}

It is theoretically possible that in our task, a sender develops an encoding strategy about the meaning of his moves, and then sticks to it during the experiment, hoping that the receiver will, in the end, figure out what the encoding strategy is. If this were the case, we would not be studying the combination of recipient design and intention recognition, but only whether receivers are able to decipher a certain encoding strategy that was previously unknown to them. So instead of trying different ways to encode information making it easier for receivers to decode it, senders would, according to this alternative theory, just use an arbitrary encoding strategy and leave it to the receiver to work out what it means.

To rule out the possibility that senders were using this strategy we used a design with 40 trials in which senders could see what receivers were doing (and hence, how the receivers had interpreted their message) and another 40 trials in which they did not get any information about the receiver (i.e., they did not see their movements or whether the trial as a whole was correct or incorrect). If the senders were systematically applying a fixed encoding strategy, then whether they know what receivers were doing or not should not influence the success of their communication.

\subsection{Method}

\subsubsection{Participants}

16 pairs of participants (aged 18-26; 23 females; 9 males) were recruited to participate. They were either offered a standard financial payment (€6 per hour) or given credits towards completing a course requirement. Subject pairs were randomly assigned to one of two conditions; starting with feedback or starting without feedback (see Table 2 below). 


\subsubsection{Equipment}

The same equipment was used as in Experiment 1, only in Experiment 2 we required our subjects to wear sound-resistant head sets and ear plugs to minimize the influence of sounds in the environment and incidental noises produced by the players. ${ }^{2}$

\subsubsection{Procedure}

After being instructed regarding the experimental tasks, participants provided their informed consent. Training was conducted to prepare them to play the TCG together. As in the first experiment, there were two training sessions before the game began to familiarize the participants with the hand-held controller, the order of events in the game, and the characteristics of the stimuli (see earlier description). Written instructions were then provided regarding the rules of the game. In all tasks, participants were instructed to perform as accurately as possible.

\subsubsection{Materials}

The game consisted of 80 trials and in all trials the receiver could not see the goal configuration that the team had to match. Players always had different shapes. $75 \%$ of the trials were 'hard' (target object orientation could not be indicated by mimicking it with the sender's shape), $25 \%$ were 'easy' (it was not necessary to indicate target orientation, or target orientation could be indicated by mimicking target orientation with the sender's shape). Table 2 shows how the feedback and no-feedback trials were distributed across the two groups.

Table 2. Distribution of conditions over the subjects

\begin{tabular}{lll}
\hline Trial Order & Group A & Group B \\
\hline Trials $1-20$ & No feedback & Feedback \\
Trials 21-60 & Feedback & No feedback \\
Trials 41-60 & Feedback & No feedback \\
Trials 61-80 & No feedback & Feedback \\
\hline
\end{tabular}

\subsection{Results}

First and foremost, we were interested in whether having access to feedback led to a higher success rate. Because the effect of feedback might be cumulative, leading to an improved success rate over the course of the experiment, a comparison of the success rates in the trials with and without feedback was not the appropriate analysis. Therefore, we performed a linear regression analysis with the probability of success of a certain trial (estimated using the data from all pairs) as dependent variable, and two independent variables: first, the order in which the trial occurred (Number of trials, with or without feedback), and second, the number of previous trials with feedback (Number of feedback-trials). If feedback was effective, the 
predictive power of the variable Number-of-feedback-trials should be significantly higher than that of Number-of-trials. Using this analysis, we could compare the effect of feedback against the baseline of a simple learning effect. It turned out that the variable Number-of-trials did not explain a significant proportion of the variance $(t=1.64, p=.102)$, whereas Number-of-feedback-trials had a positive beta weight $(\beta=0.135)$ and explained a significant proportion of the variance $(t=2.33$, $p<.05)$. These results provide clear evidence that feedback improves the dyad's performance. They also imply that the task at hand is not just a problem solving task for the sender or the receiver, but involves at least some elementary form of two-way communication between a sender and a receiver.

The second issue that we addressed was that of the processing load in both senders and receivers. First, both senders and receivers needed more planning time for the 'hard' trials than for the 'easy' trial. For receivers, this was $2.598 \mathrm{~s}$ for hard trials vs. $2.031 \mathrm{~s}$ for easy ones $(t(792)=3.19, p<.01)$. For senders the differences was $7.68 \mathrm{~s}$. for hard trials, and $5.67 \mathrm{~s}$ for the easy ones $(t(766)=6.38$, $p<.0001)$. However, part of the planning time of senders could well be due to the number of moves that they plan to make. Therefore, we performed a regression analysis with the planning time of senders as dependent variable, and the number of moves they made as well as the nature of the communicative problem (hard vs. easy) as predictors. Both factors were predictive, but the factor hard/easy (beta weight $1.24, p<.001$ ) much more so than the number of moves (beta weight 0.77 , $p=.007)$. For receivers, the number of (planned) moves did not explain any variance in the planning times (beta weight $-0.11, p=.7$ ) whereas the factor hard/easy did (beta weight $0.08, p=.007$ ) These findings suggest that the planning time of both senders and receivers are valid dependent measures that tap into the cognitive processes that we wanted to investigate.

But how do the planning times of senders and receivers compare? The mean planning time of senders was $7.18 \mathrm{~s}$, while the mean planning time of receivers was $2.46 \mathrm{~s}$. This difference is significant (paired t-test, $t(1359)=31.76, p<.0001$ ). This suggests that in communicative situations without pre-established conventions, the main cognitive load is at the sender's end of things. ${ }^{3}$ However, this comparison is confounded by the fact that receivers can already start processing while the sender is still moving his part around the board, in other words, before the clock starts ticking for the receiver. Therefore, a better way of investigating the relationship between sender and receiver planning time is to compute the (partial) correlation between them, correcting for the number of moves made by both senders and receivers (which the analaysis above showed to have a positive correlation with the planning time). If this correlation were negative, it would suggest a trade-off between cognitive effort invested by senders and receivers, in the sense that the less work the sender puts in his signal, the more work the receiver has to do, and vice versa. The partial correlation between sender and receiver planning times, 
partialing out the effect of the number of moves was $.38(p<.001),{ }^{4}$ which shows that a harder problem was more difficult for both senders and receivers. We also analysed the correlation between sender and receiver planning times without partialling out the number of moves, which resulted in a correlation of .39 $(p<.001)$. This shows that the highly significant correlation was not dependent on partialling out the number of moves. We also checked the correlation between the number of moves for senders and receivers, which was also positive $(\mathrm{r}=.410, p<.001)$. To conclude, we did not find evidence for a trade-off between cognitive effort of senders and receivers. The harder a problem is, the more moves and planning time we see for both senders and receivers.

To summarize, the results from Experiment 2 revealed that (a) feedback from receiver to sender improves the effectiveness of the communication, (b) that both senders' and receivers' planning times are sensitive to the difficulty of the communication problem, and (c) that there is no trade-off between planning time in senders and receivers, indicating that if a communication problem is hard, it is harder for both senders and receivers.

\subsection{TCG communication strategies}

To understand how our participants were able to achieve a relatively high communicative success given such limited means to communicate, we performed a detailed analysis of the first 20 individual trials for all dyads from Experiment 2 (cf. Galantucci, 2005).

For the easy trials, one predominantly used strategy was identified:

1. Move object to receiver target location

2. Rotate object to match receiver object orientation

3. Pause (longer than between the previous and following moves).

4. Move object to sender target location.

With this strategy, the overall success rate was $95 \%$ for the easy trials. Note that the longer pause is essential, as otherwise the receiver does not know which of the positions and orientations the sender object has been at is the indicated target location. In effect, pausing is used as a "deictic" device here, to first indicate where an object needed to go, and rotating (or other strategies outlined below) to indicate what had to be done with the object. More interesting were the strategies used for the hard trials. One of the reasons the hard trials presented our participants with a challenge was what we call the orientation problem: As has been already mentioned in the discussion of Experiment 1, it was not possible to mimic the orientation of a rectangle with a circle, or the orientation of a triangle with either a circle or a rectangle. This is a simple consequence of the fact that circles have only one, rectangles have two, and triangles have four possible orientations. 
To study the strategies that were used to solve the orientation problem, we inspe-cted the first 20 trials of every dyad in the experiment. During these trials, senders either had feedback all the time, or did not have feedback for any of the trials. This enabled a comparison between strategy use with and without (persistent) feedback.

In the first 20 trials, there were 13 trials in which the orientation problem occurred, leading to a total of 208 data points. The strategies that were used to solve this problem were the following, ordered by frequency of use from high to low:

A. Go to receiver target position, pause, then move the object one square in the direction that the triangle is "pointing to", or in case of the rectangle, along the axis that the rectangle is oriented along (horizontal of vertical), then move back to the receiver target position, pause, and then move to the sender target position (used 80 times, successful 60 times, $75 \%$ success rate). Strategy A is illustrated in Fig. 6 below.

B. The same strategy as described as successful for the easy trials. This strategy was used in 56 cases. This strategy obviously fails to communicate the receiver's object's orientation. Because in the hard trials the goal orientation of the receiver's object was always different from its initial orientation, it always had to be rotated. Since the target orientation was not signalled at all in this strategy, receivers did not know that their object had to be rotated, and therefore it is not surprising that the success rate of this strategy was $0 \%$.

C. Similar to $\mathrm{B}$, but trying to match the orientation of the triangle using a rectangle, used 20 times. As the triangle has four possible orientations, and the rectangle only two, this strategy maximally transmits only half the amount of information needed to identify the correct orientation of the triangle. Theoretically, this would predict maximally $50 \%$ chance of success, but in fact the strategy was only successful in 5 cases, which is $25 \%$.

D. Similar to B, but now trying to indicate orientation by selecting the path from receiver position to sender position that matches the orientation of the receiver object. This strategy is illustrated in more detail below. The strategy was used 7 times and was never successful.

E. Indicating that the triangle was "upside down" relative to its initial position by rotating the rectangle twice. This signals to the receiver that (s)he has to perform two rotation actions, which would in the case of the triangle result in it being rotated $180 \%$ degrees relative to its original orientation. This strategy was used 6 times and was successful 4 times (67\%).

F. Same as E, but now rotating the rectangle only once. This strategy was used 5 times. Although it appears to be dysfunctional, it was successful once (20\%).

G. Same as E, but now rotating the rectangle three times. This strategy was used once, and was not successful. 
F. Using a very elaborate (longer than necessary) path from starting position to receiver position to indicate the orientation vector of the receiver object. This strategy was only used once, and it was successful.

I. Finally, for 32 trials the strategy could not be identified. Of these cases, only one trial was successful (3\%).

In Table 3 below, the strategies and their frequencies are summarized.

Table 3. Used strategies and their frequencies and success rates for the orientation problem

\begin{tabular}{lccc}
\hline Strategy & Number of times used & Number of times successful & Success percentage \\
\hline A & 80 & 60 & 75 \\
B & 56 & 0 & 0 \\
C & 20 & 5 & 25 \\
D & 7 & 0 & 0 \\
E & 6 & 4 & 67 \\
F & 5 & 1 & 0 \\
G & 1 & 0 & 0 \\
H & 1 & 0 & 0 \\
I & 32 & 1 & 3 \\
\hline
\end{tabular}

The most frequently used (and most successful, if we do not count strategy $\mathrm{H}$ which was only used once) strategy $\mathrm{A}$ is illustrated in Fig. 4 below. This is an example of strategy $\mathrm{A}$ in the case of the hardest of the orientation problems, which

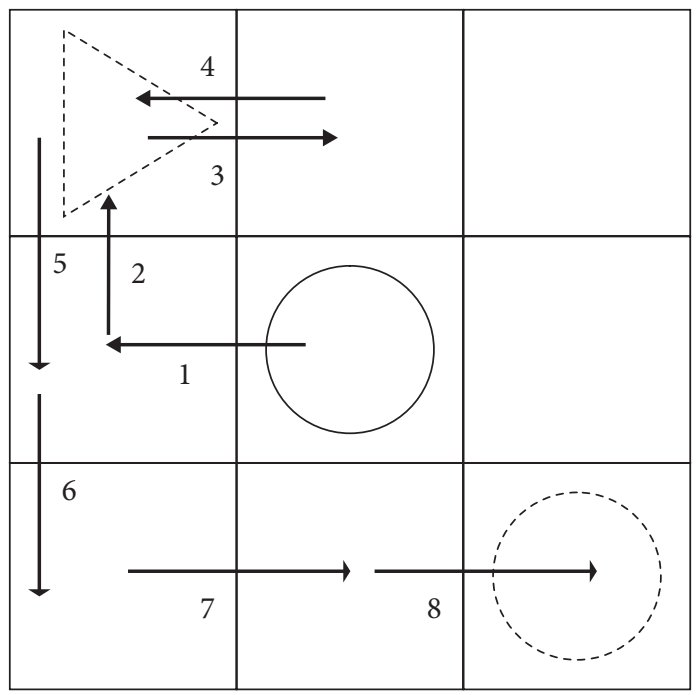

Figure 4. Example of move sequence for strategy A 
is indicating the target position and orientation of a triangle using a circle. The dotted triangle indicates the target position for the receiver object (a triangle), while the dotted circle indicates the target position for the sender object (a circle). Moves 3 and 4 are performed in order to transmit the desired orientation of the triangle. After move 2 there is a noticeable pause to indicate that the reached position is the target position.

To explain strategy D in more detail, we refer to Fig. 5 below. The dotted triangle indicates the receiver target position and orientation of the triangle. The dotted circle is the sender target position of the circle. The sender has moved his object to the receiver target location, as in the first part of strategy I. Now to move to the sender target position, the sender has two possible first moves. He can either go down or to the right. By choosing the path that starts going to the right, indicated by the solid arrows, he may be signaling to the receiver that the triangle is oriented with its point to the right. Note that this strategy only has a chance of succeeding because there is another path available as well. We show this (possible) strategy in more detail because it illustrates the problem that the receiver is faced with. She has to figure out (a) what are the choices that the sender has, (b) whether choosing a certain path is meaningful, and (c) if so, what does it mean?

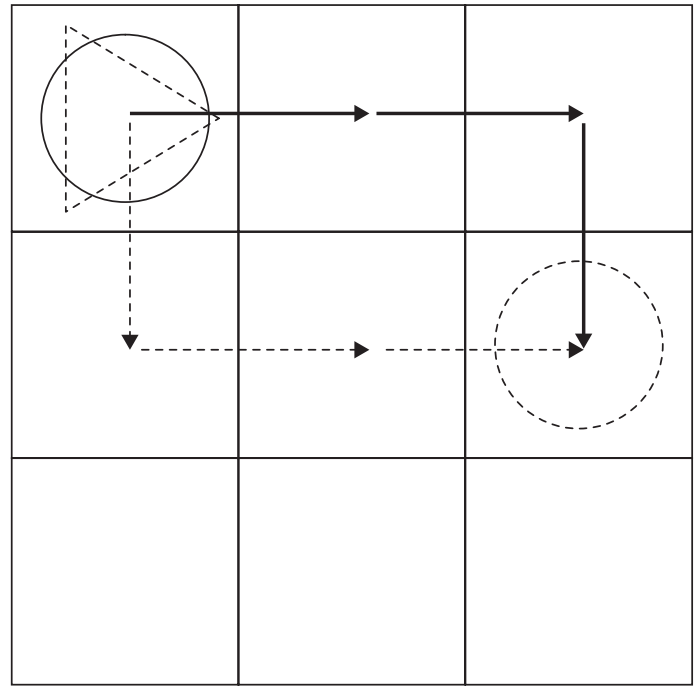

Figure 5. Example move sequence for strategy D

Strategy A, both the most used and the most successful one, reveals interesting similarities with the strategy that was predominantly and successfully used in the 
easy trials. The strategy is essentially the same, only an extra movement sequence is inserted (bracketed by pauses) to indicate the orientation of the triangle.

To summarize, we found that the successful and most frequently used signaling strategies used by senders have the following properties:

a. If there are no orientation or center problems, the sender uses his own object to signal the target location of the receiver object by moving to that location, and indicate the location by pausing at that location.

b. For the hard problems, senders re-use the strategy for the easy trials, extending the convention by inserting extra sequences to communicate the target orientation in non-conventional ways.

c. Senders separate the signaling of orientation and location by using separate motion sequences. They separate the different component messages from each other by pausing longer than between other moves (see Experiment 3 below for a statistical analysis of pause durations).

These findings shed some light on the general principles used in communication. Conventions are used whenever possible, and can be established relatively quickly. This phenomenon has also been documented in dialogue (Brennan \& Clark, 1996; Carroll, 1980; Clark \& Schaefer, 1989; Clark \& Wilkes-Gibbs, 1986; Galantucci, 2005; Schober \& Brennan, 2003). If existing conventions are not sufficient, new ones can develop, and they are likely to be built on top of the existing ones. Finally, communicators are remarkably flexible in using even highly limited channels by exploiting the degrees of freedom they have in their formulations, provided that they know that the receiver of a signal is aware of the limitations and degrees of freedom that the sender has.

The final analysis presented in this section concerns the presence of feedback or not. For half of the dyads, the first 20 trials were all with feedback, while for the other half the first 20 trials were all without feedback. If senders would indeed be helped by feedback, this would predict that senders that receive feedback would find the best solution for the orientation problem, strategy A, sooner than those without feedback. This turned out to be the case. For the dyads without feedback, the average number of previously presented trials that contained the orientation problem before the dyad started using strategy A was 10.5, while for the dyads with feedback this was $3.75(t(14)=2.415, p<.05$, two-tailed $)$. This result is consistent with the results of the regression analysis presented above. Another interesting variable is the average number of different strategies that senders used for the orientation problem. This number was not significantly different for both the feedback and no-feedback groups (no-feedback: 4.9, feedback: 4.0, $t(14)=1.59, p=.13$, two-tailed). In fact, the number of used strategies is higher for the non-feedback 
group. This indicated that even without feedback, senders do not rely on picking one strategy that they think makes sense, and then let the receiver work out what it means. Instead, even when they do not get feedback either about the success of the communication, or the interpretation by the sender (as seen in the feedback condition by the moves he makes), they try out different encoding strategies. Finally, it is important to note that the receivers in the feedback condition did not try to use extra moves to signal their level of understanding back to the receiver. The only thing they revealed to the sender was their "understanding" of the communicative intention of the sender, by making the moves that they thought were required of them. This information was sufficient for the senders to improve the effectiveness of their communicative efforts. ${ }^{5}$

\section{Experiment 3}

In a third experiment we varied the function of the behavior of the senders. Experiment 3 was aimed at studying the same behavior in either communicative or noncommunicative contexts. In one part of the experiment we asked them to move their object to the recipient's location and match the orientation of the recipient's object as well as possible, and then move to their own target location. Crucially, the recipients themselves knew the target location and orientation of their object. These are the non-communicative trials. In another part, we asked senders to signal the receiver's target location to the receiver, who, as in Experiment 1 and 2, did not know the target location or orientation of the their object. These are the communicative trials.

\subsection{Method}

\subsubsection{Participants}

16 pairs of participants (aged 18-26 years; 16 right-handed Dutch males; 16 right-handed female partners) were recruited to participate. They were either offered a standard financial payment (€6 per hour) or given credits towards completing a course requirement.

\subsubsection{Equipment}

The same equipment was used as in Experiment 1 and 2. However, in this experiment the sender was in a different room than the receiver, who played the game from another room $^{6}$ while wearing noise-resistant head phones to minimize acoustic distractions. 


\subsubsection{Procedure}

After being instructed regarding the experimental tasks, participants provided their informed consent. Training was conducted to prepare them to play the TCG together. There were three training sessions before the first part of the game to familiarize the participants with the hand-held controller, the order of events in the game, and the characteristics of the stimuli. A fourth training was performed after the first session to learn the new "version" of the game. In all tasks, participants were instructed to perform as accurately as possible.

\subsubsection{Training}

Individual Training. See Experiment 1 and 2.

Joint Training 1. See Experiment 1 and 2. For this experiment participants received only 10 Joint Training trials (all Baseline trials), because an additional joint training (Joint Training 2a) was added.

Joint Training $2 a$. In this training of 20 trials the receiver did not see the target configuration in $75 \%$ of the practice trials (Communication trials). The sender was instructed that with his shape he could try to instruct his partner on where to place the shape and which orientation it needed to be in. The other $25 \%$ of the trials were Baseline trials, in which both players could see the goal configurations and their task was to simply go to their own location and match their own orientation. Because the Communication and Baseline trials were intermixed in this training, and during the actual experiment, the transparency of the color of the shape of the receiver indicated the type of trial to participants. For Baseline trials the color of the shape of the receiver was solid red. For Communication trials the color was transparent red. This difference in transparency was highly salient and very clear to participants.

Joint Training $2 b$. In this training of 20 trials both players always saw the goal configuration, but the sender was instructed on $75 \%$ of the trials (No-Communication trials) to first move his shape to the position of the shape of the receiver and match the rotation of the shape of the receiver as well as possible. The other $25 \%$ of the trials were Baseline trials (the same as for Joint Training 2a). For Baseline trials the color of the shape of the receiver was solid red. For No-Communication trials the color was transparent red.

\subsubsection{Materials}

The game consisted of two sessions of 80 trials; 40 baseline trials and 40 trials that were either no-communication or communication trials. In both the communicative and the non-communicative conditions, some trials (10 in total per session) were 'hard' (see above at the discussion of Experiment 1 for a definition). The 16 pairs were assigned to one of two trial orders: Communication-No_Communication (eight 
pairs) or No_Communication-Communication (eight pairs) to counter-balance for order effects.

\subsection{Results}

The main purpose of Experiment 3 was to compare the same behavior occurring in either a communicative or a non-communicative context. If the planning time of senders only reflects problems they have at expressing orientation (regardless of what the aim of this expression is) then these planning times should be the same for communicative and non-communicative trials. In contrast, if the planning time of the senders also incorporate processing load for interactive processes (i.e., recipient design), then the processing load should be higher for communicative than non-communicative trials. Furthermore, given the highly limited expressive means available to the subjects, an important question is how senders use these highly limited means to inform the receiver of the intended target position and orientation.

The planning time of the sender was longer for communicative trials (Mean: $3945 \mathrm{~ms}$.) than non-communicative trials (Mean: $2705 \mathrm{~ms}$.), $t(15)=3.6, p=.003$. This difference shows that there is an additional processing load for senders in communicative than non-communicative trials, which we would explain as reflecting additional interactive processes related to recipient design.

After an extensive qualitative inspection of the motion sequences that senders used in the TCG (see previous section), it appeared that senders first moved their object to the receiver's location to indicate the goal position, using timing to indicate which of the many positions that the sender's object had reached was the actual receiver's position. It appeared that when the sender's object was on the (receiver's) target position, it was held still a little longer than when it was on other positions. To test whether this is a statistically reliable effect, we compared how long the sender's object held still on the intended receiver's position (if the sender did move at all to that position), and how long the sender held still on all other positions. We excluded the first and the last position of movement sequences from this analysis, as they are contaminated by the fact that they mark the beginning and end of the entire trial. If senders use timing (i.e., longer pausing) to indicate that a certain position is the goal position, there should be (a) longer holding times at receiver's goal locations than at other locations, and (b) this effect should be larger for communicative trials than for non-communicative trials. The results of this analysis (see Fig. 6) are clear. First, senders hold their object for a longer duration in target positions than in non-target positions $(\mathrm{F}(1,20)=83.4, p<.001)$, and second, this effect is much larger for the communicative condition: the interaction for pause times between the factors communicativeness (yes vs. no) and position 
(target vs. non-target) is significant $(F(1,20)=19.3, p<.001)$. Note that target positions are often points where the direction of the motion sequence is reversed, which explains why target positions have longer average pause times in the noncommunicative condition as well.

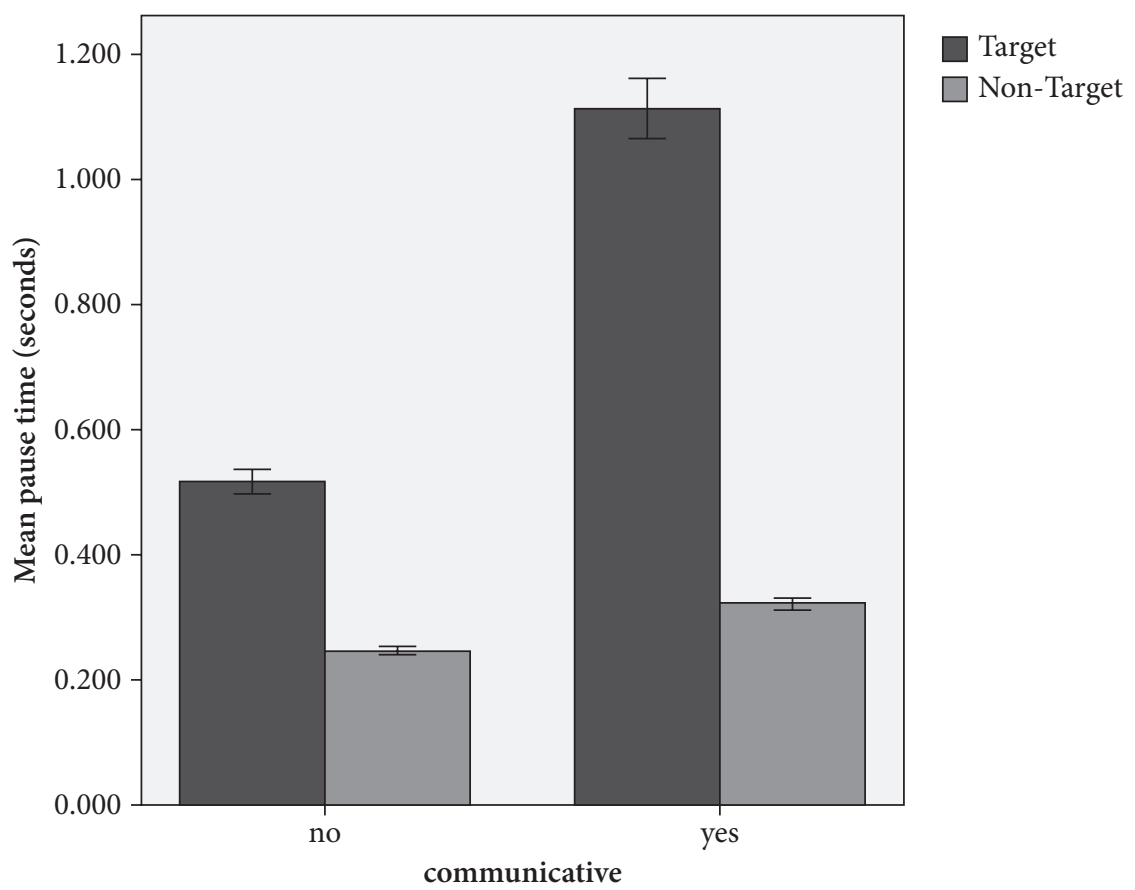

Figure 6. Mean pause times in senders' moves

\section{Summary and conclusions}

Communication is more than just data transmission from senders to receivers, and some theoreticians (e.g., Levinson, 1995, 2006) have claimed that humans have a specialized kind of cognitive processing to deal with the complexities of intention recognition and recipient design. We set out to directly explore these cognitive processes in the laboratory, in isolation from the conventional linguistic system that usually accompanies it. We found that most subjects were able to communicate successfully without any pre-established conventions (cf. Galantucci, 2005), and that the effectiveness of the communication increases when senders have access to feedback from the receiver, showing that the task is truly interactional. We believe that these findings provide some support for the 
notion of a specialized communicative intelligence, for the following reasons. First, the problem that the participants were facing is not a classical "puzzle" with a specific sought-after "solution". Rather, the task put the participants in a communicative context and required of them to produce communicative behavior, not solve a cognitive conundrum. Second, Experiment 3 shows that an essential component of the resulting communicative behavior was to introduce differences in inter-move timing in the order of a few hundred milliseconds, which is a level of precision in the timing of behavior that is normally outside of conscious control. However, there is an alternative explanation for our findings, namely that our participants were not using specialized communicative skills, but rather were invoking general cognitive (problem solving) capacities to perform so well on our task. We concede that this alternative explanation cannot be ruled out by our findings. That would ideally require experimenting with individuals with high problem solving skills on the one hand, and limited communicative skills on the other. Perhaps further experiments with participants with high-functioning autism-spectrum disorders (e.g., Asperger syndrome) could resolve this issue. If individuals from such populations would show an ability to solve complex cognitive problems of a similar cognitive complexity, but not the TCG, this would provide conclusive support for the existance of specialized communicative processing.

The task appears to be a valid tool for estimating the cognitive load associated with recipient design in senders and intention recognition in receivers simultaneously. The strongest evidence for the validity of our task is that the harder the communicative problem is, the more planning time is needed by both the sender and the receiver. This extra planning time was mainly caused by the nature of the communicative problem, and much less by the number of moves that were performed per trial.

This task allows us to explore the "interactional intelligence" which is typical for human communication in a context which strips away the complex conventions that are normally erected on that foundation, and which thus obscure it. The task, or variants thereof, could be used to address a host of scientific questions beyond the ones addressed in this study. It could be used to assess the underlying communicative capabilities in a variety of genetic and neurological disorders (e.g., Autism Spectrum Disorders, Williams syndrome, Down's syndrome, aphasia etc.) independent of the varying linguistic competence of the investigated subjects. The task might also be adapted to investigate the communicative capabilities in other social vertebrates, which lack an arbitrary conventional sign system, and to explore the development of interactive capabilities in human infancy. Furthermore, the task could also be used to measure other dependent variables such as neurophysiological signals and eye-tracking data, and thus promises to open up the nature of human interactive intelligence to many different kinds of experimental probe. 
It is important to stress that although the task used in our experiment differs from everyday communication (in which conventions are abundant), the cognitive process that we try to focus on in our experiments is fundamental for 'ordinary' communication. Even well articulated linguistic utterances are pervasively ambiguous, as illustrated by the infamous and frequently used definite reference (e.g., "when is the meeting?"). Only the combination of recipient design in senders and intention recognition in receivers enables communicators to be effective.

Finally, we found that people are remarkably effective in using whatever limited means of communication they have. While moving around their object on the grid, senders in our study systematically used manner (which, in the context of the task we used, was expressed by timing) as a nonverbal diacritic to indicate a certain position, thereby also assisting receivers in parsing the sequence of behavioral events into meaningful sub-units. As slowing down at certain positions is not instrumental for the functional goal of the sender (i.e., moving an object to a location), receivers can infer that the slowing down performs a communicative function. In this case, the inferred function is a deictic one: it singles out a certain position from other possible positions. This type of inference is similar to the finding by Gergely, Bekkering \& Király (2002) that children who observe an adult switching on a light with their head will only imitate the use of the head if the hands of the adult were unrestrained. When the use of the head in that situation is not functionally motivated (or 'rational', see Gergely \& Csibra, 2003), it is interpreted as providing information in the sense of Bateson's (1972) famous definition: "information is a difference that makes a difference" (p. 453).

The insight that cooperative interaction is subject to this kind of meansends reasoning, so that deviations from the functionally optimal strategy carry communicative load, forms the central idea in Grice's (1975) other important theory, concerning the maxims of cooperative interaction. Thus, the use of nonfunctional behavior for communicative purposes is also very common in linguistic communication. For example, in referring to third persons, providing more information than is strictly necessary for resolving the reference communicates additional information to the listener. If someone refers to a colleague as "Professor William F. Smith", where a simple "Bill" would have sufficed, the apparent redundancy in the reference signals the presence of additional communicative intentions (see Enfield \& Stivers (2007) for examples from different languages, and Levinson (2000) for the general principle involved). The finding that this same principle is used in both tacit nonverbal communication and verbal interaction using all the rich resources of linguistic conventions provides additional evidence for the existence of a specialized cognitive infrastructure that is dedicated to communication. 


\section{Acknowledgments}

This work has been completed in the context of the European Union Integrated Project JAST (Joint Action Science and Technology), grant FP6-IST2-003747.

\section{Notes}

1. Interestingly, of all the trials that were the first to occur in the experimental sequence, $42 \%$ was successful, which is still above chance.

2. We first ran two subject pairs that were subsequently excluded from the experiment: one pair because of their extremely poor performance which turned out to be due to a misunderstanding of the instruction, and another pair was excluded because they used auditory means of communication: they were "rotating" the circle, which had no visual effect but communicated by transmitting multiple clicking sounds. These problems were addressed by (a) creating a clearer instruction and (b) requiring the participants to wear ear-plugs and closed noise-resistant headphones to prevent any form of auditory communication.

3. Separate analysis for successful and non-successful trials resulted in the same pattern of results for both groups.

4. When we analyzed successful and non-successful trials separately, we found the same pattern of results for both groups of trials.

5. We are indebted to an anonymous reviewer who pointed out to us the interesting possibility of receivers using their moves to send "meta-information" back to the senders.

6. The reason for moving one of the participants to another room was that we wanted to extend the paradigm such that we could put one of the participants in an MR scanner.

\section{References}

Anderson, A.H., Bader, M., Gurman Bard, E., Boyle, E.A., Doherty-Sneddon, G., Garrod, S.C., et al. (1991). The HCRC Map Task Corpus. Language \& Speech, 34(4), 351-366.

Bateson, G. (1972). Steps to an Ecology of Mind. New York: Ballantine.

Brennan, S.E., \& Clark, H.H. (1996). Conceptual Pacts and Lexical Choice in Conversation. Journal of Experimental Psychology: Learning, Memory, and Cognition, 22(6), 1482-1493.

Carroll, J.M. (1980). Naming and describing in social communication. Language and Speech, 23, 309-322.

Clark, H.H., \& Marshall, C.R. (1981). Definite reference and mutual knowledge. In A.K. Joshe, B.L. Webber \& I.A. Sag (Eds.), Elements of discourse understanding (pp. 10-63). Cambridge, U.K.: Cambridge University Press.

Clark, H.H., \& Schaefer, E.F. (1989). Contributing to discourse. Cognitive Science, 13, 259-294.

Clark, H.H., \& Wilkes-Gibbs, D. (1986). Referring as a collaborative process. Cognition, 22, 1-39. 
De Ruiter, J.P., Noordzij, M., Newman-Norlund, S., Hagoort, P., \& Toni, I. (2007). On the origin of intentions. In Y. Rossetti, P. Haggard \& M. Kawato (Eds.), Sensorimotor foundations of higher cognition (pp. 593-610). Oxford: Oxford University Press.

De Ruiter, J.P., Rossignol, S., Vuurpijl, L., Cunningham, D.C., \& Levelt, W.J.M. (2003). SLOT: A research platform for investigating multimodal communication. Behavior Research Methods, Instruments, \& Computers, 35(3), 408-419.

Enfield, N.J. (2003). The definition of what-d'you-call-it. Journal of Pragmatics, 31, 101-117.

Enfield, N.J., \& Stivers, T. (Eds.). (2007). Person reference in interaction: linguistic, cultural and social perspectives. Cambridge: Cambridge University Press.

Galantucci, B. (2005). An experimental study of the emergence of human communication systems. Cognitive Science, 25(5), 737-767.

Garrod, S., \& Anderson, A. (1987). Saying what you mean in dialogue: A study in conceptual and semantic co-ordination. Cognition, 27, 181-218.

Garrod, S., Fay, N., Lee, J., Oberlander, J., \& Macleod, T. (2007). Foundations of Representation: Where Might Graphical Symbol Systems Come From. Cognitive Science, 31(6), 961-987.

Gergely, G., Bekkering, H., \& Király, I. (2002). Rational imitation in preverbal infants. Nature, 415, 755.

Gergely, G., \& Csibra, G. (2003). Teleological reasoning in finance: the naÏve theory of rational action. Trends in Cognitive Science, 7(7), 287-292.

Goldin-Meadow, S., Gelman, S.A., \& Mylander, C. (2005). Expressing generic concepts with and without a language model. Cognition, 96(2), 109-126.

Grice, H.P. (1957). Meaning. Philosophical Review, 66, 377-388.

Grice, H.P. (1975). Logic and conversation. In P. Cole \& J.L. Morgan (Eds.), Syntax and semantic 3: Speech acts (pp. 41-58). New York: Academic press.

Healey, P.G.T., Swoboda, N., Umata, I., \& King, J. (2007). Graphical Language Games: Interactional constraints on representational form. Cognitive Science, 31, 285-309.

Levelt, W.J.M. (1989). Speaking: from intention to articulation. Cambridge, Massachusetts: The MIT Press.

Levinson, S.C. (1983). Pragmatics. Cambridge: Cambridge University Press.

Levinson, S.C. (1995). Interaction biases in human thinking. In E.N. Goody (Ed.), Social intelligence and interaction (pp. 221-260). Cambridge: Cambridge University Press.

Levinson, S.C. (2000). Presumptive Meanings; The Theory of Generalized Conversational Implicature. Cambridge, Massachusetts: The MIT Press.

Levinson, S.C. (2006). On the human “interactional engine”. In N.J. Enfield \& S.C. Levinson (Eds.), Roots of Human Sociality: Culture Cognition, and Interaction. London: Berg.

Lewis, D.K. (1968). Convention: A philosophical study. Cambridge, MA.: Harvard University Press.

Repp, B.H., \& Liberman, A.M. (1987). Phonetic category boundaries are flexible. In S. Harnad (Ed.), Categorical perception. Cambridge: Cambridge University Press.

Sacks, H., \& Schegloff, E.A. (1979). Two preferences in the organization of reference to persons in conversation and their interaction. In G. Psathas (Ed.), Everyday language: Studies in ethnomethodology (pp. 15-21). New York: Irvington.

Schegloff, E.A. (1988). Presequences and indirection; applying speech act theory to ordinary conversation. Journal of Pragmatics, 12, 55-62.

Schelling, T. (1960). The strategy of conflict. Cambridge, Mass.: Cambridge University Press.

Schiffer, S. (1972). Meaning. Oxford: Clarendon Press. 
Schober, M.F., \& Brennan, S.E. (2003). Processes of Interactive Spoken Discourse: The Role of the Partner. In A.C. Graesser, M.A. Gernsbacher \& S.R. Goldman (Eds.), Handbook of Discourse Processes (pp. 123-164). Mahwah, New Jersey: Lawrence Erlbaum.

Shannon, C.E. (1948). A mathematical theory of communication. Bell System Technical Journal, 27, 379-423,623-656.

Steels, L. (2006). Experiments on the emergence of human communication. Trends in Cognitive Science, 10, 347-349.

Tomasello, M., Carpenter, M., Call, J., Behne, T., \& Moll, H. (2005). Understanding and sharing intentions: The origins of cultural cognition. Behavioral and Brain Sciences, 28(5), 675-691. Wharton, T. (2003). Natural Pragmatics and Natural Codes. Mind \& Language, 18(5), 447-448. 